\title{
Frequency-Domain Set-Membership Filtering and Its Applications
}

\author{
Li Guo and Yih-Fang Huang, Fellow, IEEE
}

\begin{abstract}
Frequency-domain adaptive filtering is appealing in many applications, particularly channel equalization. This paper presents frequency-domain set-membership filtering (F-SMF) and derives adaptive algorithms for F-SMF. The F-SMF is employed to design single-carrier frequency-domain equalizer (SC-FDE). With an unconventional parameter-dependent error-bound specification, an F-SMF algorithm is derived and shown to provide superior performance with sparse updates of parameter estimates. Exploring the feature of sparse updates, we present an innovative parallel adaptive architecture that shares the updating processors and that finds natural appeal in frequency-domain diversity combining and equalization for very dispersive fading channels like those found in broadband wireless communications.
\end{abstract}

Index Terms-Adaptive frequency-domain equalization, frequency-domain filters, parallel adaptive subsystems, set-membership filtering, updator-sharing.

\section{INTRODUCTION}

$\mathbf{S}$ ET-MEMBERSHIP filtering (SMF) and a class of recursive algorithms (see, e.g.,[1]-[8]) were developed as an alternative to conventional filtering approaches such as recursive least-squares (RLS) and least-mean-square (LMS) algorithms. Generally, with an a priori error-bound specification, SMF algorithms seek filter weights such that the worst case error is bounded. Numerous investigations (see, e.g., [1]-[3], [6]) have shown those algorithms' attractive features, highlighted by data-dependent selective update (of parameter estimates). The selective updates result from optimizing the step-size at each data point, which provide a convenient performance-complexity tradeoff. Unlike the traditional algorithms (e.g., LMS and RLS) that continually update parameter estimates regardless of the benefit of those updates, SMF algorithms can "intelligently" evaluate the incoming data on their benefit to improve the quality of the estimates accordingly. Consequently, most SMF algorithms exhibit superior convergence and tracking performance [1], [6], compared to traditional LS

Manuscript received June 11, 2005; revised May 3, 2006. The associate editor coordinating the review of this paper and approving it for publication was Dr. Vitor Heloiz Nascimento. This work was supported in part by the National Science Foundation by Grant EEC02-03366, by the U.S. Department of the Army under Contract DAAD 16-02-C-0057-P1, and by the Indiana 21st Century Fund for Research and Technology. This work was completed while L. Guo was at the University of Notre Dame pursuing the Ph.D. degree.

L. Guo was with the Department of Electrical Engineering, University of Notre Dame, Notre Dame, IN 46556 USA. He is now with Navini Networks, Richardson, TX 75082 USA (e-mail: 1guo@navini.com).

Y.-F. Huang is with the Department of Electrical Engineering, University of Notre Dame, Notre Dame, IN 46556 USA (e-mail: huang@nd.edu).

Color versions of figures available online at http://ieeexplore.ieee.org.

Digital Object Identifier 10.1109/TSP.2006.888890 and LMS algorithms. These algorithms have been employed for a variety of applications that include speech coding [9], adaptive equalization [8], [10], mitigation of multiple access interference in wireless communications [11], etc.

To date, investigations on SMF have mostly been limited to time-domain symbol-wise filtering. Implementation of time-domain adaptive FIR filters may be prohibitively complex in some applications such as channel equalization in broadband wireless communications [12], [13], and echo cancellation [14]-[16]. In comparison, a frequency-domain (FD) filter usually enjoys lower implementational complexity [17]. In particular, the computational complexity of FD filter is in the order of logarithm of the filter length, while that of time-domain (TD) filter is at least linearly proportional to the filter length. The extension of SMF theory to frequency domain is therefore of much interest. This paper presents a framework for frequency-domain set-membership filtering (F-SMF). Similarly to its time-domain counterpart (which is termed T-SMF in this paper), the F-SMF problem is formulated on the basis of a bounded vector-error specification. An error-bound specification is imposed on each frequency bin, which can be varied to assure uniform convergence performance across all frequency bins. Recursive algorithms for F-SMF are then derived. These algorithms are shown to offer improved performance of faster convergence and lower steady-state error with low computational complexity, and are shown to be applicable to single-carrier frequency-domain equalizer (SC-FDE) which is suitable for broadband wireless communications. Accordingly, a framework of set-membership frequency-domain equalization (SM-FDE) is presented along with some sufficient and necessary conditions for the existence of feasible equalizers.

A fundamental challenge in SMF is the need to choose an error bound a priori, which may critically affect the performance. Practically, it is often difficult to choose an error bound accurately. This problem is even more pronounced when F-SMF algorithms are employed for FDE. The reason is that each symbol is transformed into a number of bins and it is desirable to choose different error bounds for different bins. To resolve this problem, we derive an F-SMF adaptive algorithm for FDE with parameter-dependent error bounds. Unlike most of the existing SMF algorithms, see, e.g., [1], [2], [4], [5], this algorithm needs no a priori specification on the error bound. Thus it has less risk of overbounding and underbounding while enjoying all the advantages of conventional SMF algorithms. Further, the proposed algorithm can track the time-variant "true" error bound.

Exploring the selective-update feature of SMF algorithms, an updator-sharing scheme can be constructed for F-SMF, similarly to its time-domain counterpart [10]. The U-SHAPE adap- 


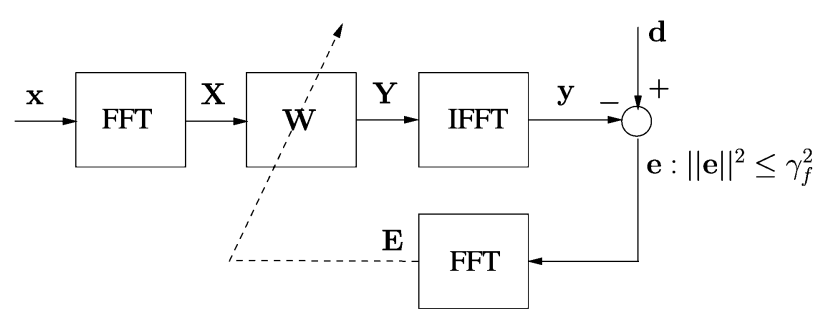

Fig. 1. Frequency-domain set-membership filtering.

tive filter architecture [10] can potentially offer significant hardware savings. This adaptive filter structure is particularly suitable for broadband wireless receivers with multiple antennas and FDE, e.g., diversity combining and equalization. This proposed scheme can drastically reduce the hardware requirement (up to $89 \%$, according to our experience) with little performance degradation.

Notation: Lowercase (uppercase) letters represent time-domain (frequency-domain) quantities. Boldface denotes vectors while a tilde sign denotes matrices. Superscripts ${ }^{*},{ }^{T},{ }^{H}$ represent complex conjugate, transpose, and Hermitian operations, respectively. Operators $\circ$ and $\div$ denote element-by-element multiplication and division of two vectors, respectively. $\operatorname{diag}\{\mathbf{x}\}$ is a diagonal matrix with vector $\mathbf{x}$ on its diagonal and $\tilde{F}$ denotes the $N \times N$ FFT matrix with elements $\{\tilde{F}\}_{m, n=0}^{m, n=N-1}=(1 / \sqrt{N}) e^{-i(2 \pi / N) m n}$.

\section{FREQUENCY-DOMAIN SET-MEMBERSHIP FILTERING}

\section{A. Frequency-Domain Filtering}

Consider a general linear-in-parameter frequency-domain (FD) filter with input vector $\mathbf{x} \in \mathbb{C}^{N \times 1}$ and desired output vector $\mathbf{d} \in \mathbb{C}^{N \times 1}$, as shown in Fig. 1. The input vector $\mathbf{x}$ is first transformed through $N$-point FFT into an FD vector $\mathbf{X} \in \mathbb{C}^{N \times 1}$. The filtering is conducted bin-by-bin in the frequency domain. Assume the filter coefficient vector in the frequency domain is $\mathbf{W} \in \mathbb{C}^{N \times 1}$. Thus, the FD filter output is $\mathbf{Y}(\mathbf{W})=\mathbf{W} \circ \mathbf{X}$. Then the filter output is transformed back into the time domain through IFFT, resulting in the time-domain (TD) filter output $\mathbf{y}(\mathbf{W})=\tilde{F}^{H} \mathbf{W} \circ \mathbf{X}$. The TD filter error is defined as $\mathbf{e}(\mathbf{W})=\mathbf{d}-\mathbf{y}(\mathbf{W})$, which, in the frequency domain, is $\mathbf{E}(\mathbf{W})=\mathbf{D}-\mathbf{Y}(\mathbf{W})$, where $\mathbf{D} \in \mathbb{C}^{N \times 1}$ is the FFT of $\mathbf{d}$. The FD filters are particularly appealing in those applications where computational complexity is prohibitively high for TD filters. Conventional FD filtering schemes are simply converted from the TD LS or LMS approaches, which select parameter vector $\mathrm{W}$ so as to minimize the time-average or ensemble average of the squared error.

\section{B. Formulation of F-SMF}

The frequency-domain set-membership filtering (F-SMF) is formulated with a bounded error-vector specification. To begin with, define the model space $\mathcal{S} \in \mathbb{C}^{N} \times \mathbb{C}^{N}$, which is the set of all possible input vector-desired output vector pairs $(\mathbf{x}, \mathbf{d})$. An equivalent FD model space $\mathcal{S}_{f}$ is defined as the set of all possible
FD input vector-desired output vector pairs $(\mathbf{X}, \mathbf{D})$. Obviously, $\mathcal{S}_{f}=\tilde{T} \mathcal{S}$, where the linear transformation matrix $\tilde{T}$ is

$$
\tilde{T}=\left(\begin{array}{cc}
\tilde{F} & 0 \\
0 & \tilde{F}
\end{array}\right)
$$

With the bounded error-vector specification $\gamma_{f}$, the objective of F-SMF is to find a parameter vector $\mathbf{W}$ such that

$$
\|\mathbf{e}(\mathbf{W})\|^{2} \leq \gamma_{f}^{2} \quad \text { for all }(\mathbf{x}, \mathbf{d}) \in \mathcal{S}
$$

where $\mathbf{e}(\mathbf{W})$ is the TD filter error, i.e., the difference between the desired output and the filter output. By the property of IFFT $\|\mathbf{e}(\mathbf{W})\|^{2}=\|\mathbf{E}(\mathbf{W})\|^{2}$, criterion (1) is equivalent to:

$$
\|\mathbf{E}(\mathbf{W})\|^{2} \leq \gamma_{f}^{2} \quad \text { for all }(\mathbf{X}, \mathbf{D}) \in \mathcal{S}_{f} .
$$

In the sequel, (2) will be used as the objective of F-SMF. The solution to (2) is, in general, a set of parameter vectors. Any member of this set is a valid F-SMF that satisfies the bounded error-vector specification. Each pair of $(\mathbf{X}, \mathbf{D}) \in \mathcal{S}_{f}$ induces an instantaneous set of parameter estimates, termed constraint set, given by

$$
\mathcal{H}(\mathbf{X}, \mathbf{D})=\left\{\mathbf{W} \in \mathbb{C}^{N \times 1}:\|\mathbf{D}-\mathbf{W} \circ \mathbf{X}\|^{2} \leq \gamma_{f}^{2}\right\}
$$

The solution of F-SMF, referred to as the feasibility set, is the intersection of all the constraint sets due to all possible data pairs in $\mathcal{S}_{f}$. This set is given by

$$
\mathcal{W} \triangleq \bigcap_{\left(\mathbf{X}, \mathbf{D} \in \mathcal{S}_{f}\right)}\left\{\mathbf{W} \in \mathbb{C}^{N \times 1}:\|\mathbf{D}-\mathbf{W} \circ \mathbf{X}\|^{2} \leq \gamma_{f}^{2}\right\} \text {. }
$$

The feasibility set and the constraint set of F-SMF have many properties similar to their TD counterparts (i.e., T-SMF), except that the constraint set of F-SMF is the interior of a hyperellipsoid while that of the T-SMF is the region between two parallel hyperplanes [6].

As is the case in T-SMF, the feasibility set of F-SMF may be empty when the error-bound specification is too stringent and is smaller than the smallest feasible specification. This can be circumvented by increasing $\gamma_{f}$ or reducing $\mathcal{S}_{f}$ or even increasing the filter length. If $\mathcal{S}_{f}$ is known a priori, we can design an F-SMF given the choices of $N$ and $\gamma_{f}$. However, in practice, the model space is often not completely known and/or time-varying. Thus adaptive algorithms are needed to design an effective F-SMF.

\section{Adaptive F-SMF Algorithms}

To derive an adaptive F-SMF algorithm, we modify the objective of F-SMF by replacing the scalar specification $\gamma_{f}$ with a vector specification, namely $\Gamma_{f}=\left[\gamma_{0}, \ldots, \gamma_{N-1}\right]^{T}, \Gamma_{f}^{T} \Gamma_{f}=$ $\gamma_{f}^{2}$, where $\gamma_{k} \geq 0, k=0,1, \ldots, N-1$, applies to the $k$-th frequency bin. Thus the modified F-SMF objective is to find $\mathbf{W}$ such that the error in each frequency bin $k$ satisfies

$$
\left|E_{k}\right|^{2}=\left|D_{k}-W_{k} X_{k}\right|^{2} \leq \gamma_{k}^{2} \quad(\mathbf{X}, \mathbf{D}) \in \mathcal{S}_{f}
$$


It will be shown that this modification would result in adaptive F-SMF algorithms with a simple structure. With different errorbound specifications for different bins, the resulting adaptive algorithm would potentially have more uniform performance among all frequency bins. Further, this modification can facilitate an updator-sharing scheme which will be presented later.

Assume that a sequence of $\left(\mathbf{X}_{n}, \mathbf{D}_{n}\right) \in \mathcal{S}_{f}$ is available for "training." The constraint set due to the $n$th observed data pair is

$$
\mathcal{H}_{n}=\bigcap_{k=0}^{N-1}\left\{\mathbf{W} \in \mathbb{C}^{N \times 1}:\left|D_{n, k}-W_{n, k} X_{n, k}\right|^{2} \leq \gamma_{k}^{2}\right\}
$$

which is the region enclosed by a polyhedron described by $\left|D_{n, k}-W_{n, k} X_{n, k}\right|^{2}=\gamma_{k}^{2}$ with $k=0, \ldots, N-1$. Given the observations $\left(\mathbf{X}_{i}, \mathbf{D}_{i}\right)_{i=1}^{n}$, define the membership set $\Psi_{n}$ as the intersection of the constraint sets, up to and including the present instant, i.e., $\Psi_{n}=\bigcap_{i=1}^{n} \mathcal{H}_{i}$. Note that $\Psi_{n}$ is a superset of the feasibility set and is the minimal set estimate given the observations $\left(\mathbf{X}_{i}, \mathbf{D}_{i}\right)_{i=1}^{n}$.

An objective of adaptive SMF is to track the membership set at each instant. One approach is to form a sequence of sets that converge to the feasibility set by tracking $\Psi_{n}$ with a point-wise approach [6]. We now derive an adaptive algorithm, referred to as F-SM-NLMS, from a point-wise approach based on the principle of minimal disturbance. Given the previous parameter estimate $\mathbf{W}_{n-1}$, the new estimate $\mathbf{W}_{n}$ is found by

$$
\mathbf{W}_{n}=\arg \min _{\mathbf{W} \in \mathcal{H}_{n}}\left\|\mathbf{W}-\mathbf{W}_{n-1}\right\|^{2} .
$$

The update equation for the resulting F-SM-NLMS algorithm is

$$
\mathbf{W}_{n}=\mathbf{W}_{n-1}+\tilde{\mu}_{n} \mathbf{E}_{n} \div \mathbf{X}_{n}
$$

where $\tilde{\mu}_{n}$ is the adaptive step matrix given by

$$
\tilde{\mu}_{n}=\operatorname{diag}\left\{\left[\mu_{n, 0}, \ldots, \mu_{n, N-1}\right]^{T}\right\}
$$

with

$$
\mu_{n, k}= \begin{cases}0, & \left|E_{n, k}\right|^{2} \leq \gamma_{k}^{2} \\ 1-\frac{\gamma_{k}}{\left|E_{n, k}\right|}, & \left|E_{n, k}\right|^{2}>\gamma_{k}^{2}\end{cases}
$$

In some cases, the output of recursion (8) exhibits some fluctuations. To smooth the output, we could replace $\mathbf{X}_{n}$ with $\mathbf{P}_{n} \div \mathbf{X}_{n}^{*}$, where $\mathbf{P}_{n}=\left[p_{n, 0}, \ldots, p_{n, N-1}\right]^{T}$. Each element in $\mathbf{P}_{n}$ can be computed recursively by [18]

$$
\begin{gathered}
p_{n, k}=\lambda p_{n-1, k}+(1-\lambda)\left|X_{n, k}\right|^{2} \\
p_{0, k}=\delta_{k}, \quad k=0,1, \ldots, N-1
\end{gathered}
$$

where $\lambda \in(0,1]$ is usually referred to as the smoothing factor and $\delta_{k}$ is a small positive constant.

A frequency-domain set-membership algorithm was presented in [19]. The F-SM-NLMS algorithm proposed here is different from the algorithm of [19] in the following aspects: 1) the algorithm of [19] was derived with a total energy constraint (on the noise), as opposed to instantaneous constraint used in this paper; thus the feasibility set of our F-SM-NLMS algorithm is a subset of that of the algorithm in [19] as long as

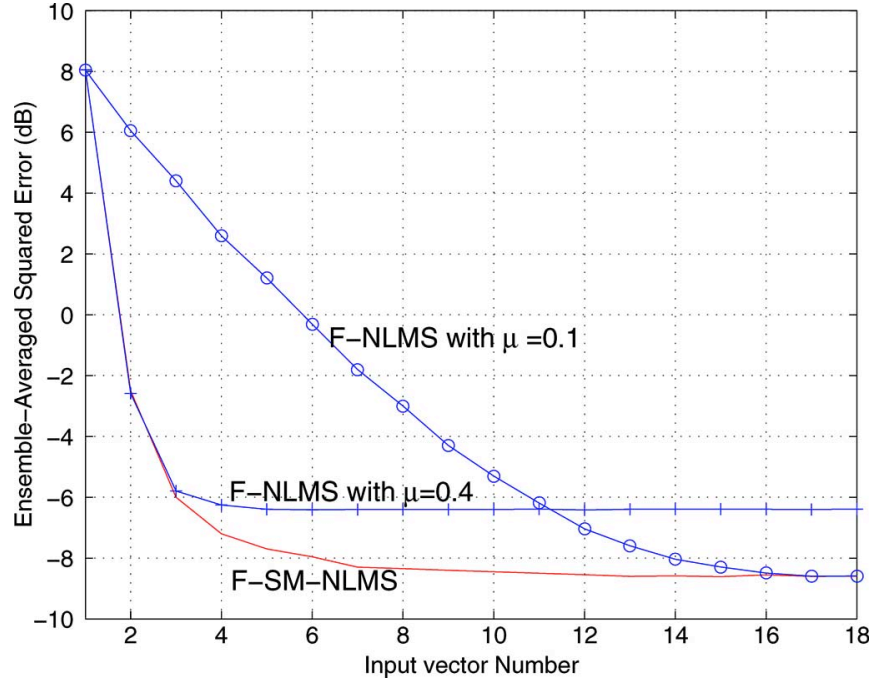

Fig. 2. MSE comparison between F-SM-NLMS and F-NLMS.

$\sum_{k=0}^{N-1} \gamma_{k}^{2} \leq \gamma_{\mathrm{en}}^{2}$, where $\gamma_{\mathrm{en}}^{2}$ is the total energy constraint specification of the algorithm in [19]; 2) the F-SMNLMS algorithm uses different step-sizes for the update in different bins and allows for independent update of different bins, which is not the case for the algorithm in [19]; 3) based on our simulation experience, F-SM-NLMS seems to always have a faster convergence rate than the algorithm of [19]; and 4) F-SM-NLMS algorithm has lower computational complexity.

\section{Performance of F-SMF}

It is clear that one of the significant advantages of F-SMNLMS over F-NLMS is that its step-size (for updating parameter estimates) varies in accordance with the received data, (10). Since the step-size can be zero, F-SM-NLMS updates selectively, just like all SMF algorithms (see, e.g., [1] and [6]).

Fig. 2 shows the mean-square error (MSE) learning curves of identifying a 100-tap complex-coefficient filter with additive white Gaussian noise (AWGN) for both F-SM-NLMS and F-NLMS algorithms. The filter to be identified is a truncated FIR microwave radio channel obtained from actual field measurements [20]. This simulation can also be viewed as an example of channel estimation in fixed wireless communications. We implemented smoothing, (11)-(12), in both F-SM-NLMS and F-NLMS algorithms. In F-NLMS, the smoothing is used to ensure that all bins have the same convergence rate. The SNR is $10 \mathrm{~dB}$ and the input vector is a correlated Gaussian signal obtained by passing a white Gaussian signal through a first-order low-pass filter with an eigenvalue spread of 80 . A large eigenvalue spread is used for an investigation on the robustness issues. The bound $\gamma_{k}$ for each bin is set to be $\sqrt{5 \sigma_{v}^{2}}\left(\sigma_{v}^{2}\right.$ is the noise variance). The reason for choosing same error bound for different bins is that the output noise is white. The simulation result of F-SM-NLMS shows a faster convergence and lower steady-state error than F-NLMS. This is consistent with the results obtained for time-domain adaptive filtering using SM-NLMS and comparing to NLMS [6]. Further examination on the performance of frequency-domain adaptive equalizers using F-SM-NLMS 


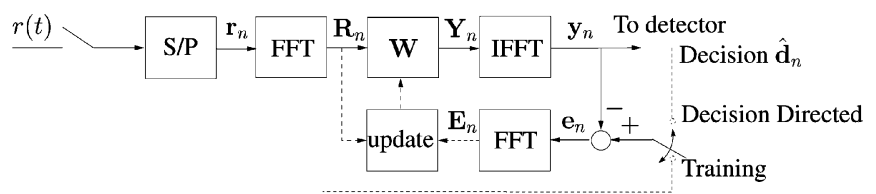

Fig. 3. Receiver with single-carrier frequency-domain equalization.

and F-NLMS is given in the next section where superior performance of F-SM-NLMS is shown.

\section{SET-MEMBERShIP FREQUENCY-DOMAIN EQUALIZATION}

In this section, we present an application of F-SMF to single-carrier frequency-domain equalization (SC-FDE) [12] which is considered a viable technique for broadband wireless communications. Compared with multicarrier communication employing OFDM (orthogonal frequency division multiplexing), SC-FDE offers a number of significant advantages that include, e.g., lower peak-to-average ratio of transmitted power, robustness with respect to phase noise and frequency offset, ability to explore multipath diversity, etc. (see, e.g., [12], [21]-[23]). It is one of the optional techniques for $2-11 \mathrm{GHz}$ air interface specification in IEEE 802.16 [24].

\section{A. Frequency-Domain Equalization}

Consider a single-carrier block transmission with a cyclic prefix (CP) of $L$ symbols (where $L$ is the channel order), and the transmitted data sequence is broken into blocks (i.e., vectors) of $N$ symbols. The CP ensures that the block has a cyclic property at the receiver and it precedes each block to be transmitted. Frequency-domain equalization, Fig. 3, is employed at the receiver. After the $\mathrm{CP}$ is discarded, the $n$th received data vector is

$$
\mathbf{r}_{n}=\tilde{C}_{h} \mathbf{d}_{n}+\mathbf{v}_{n}
$$

where the noise vector is

$$
\mathbf{v}_{n}=\left[v_{n, 0}, v_{n, 1}, \ldots, v_{n, N-1}\right]^{T}
$$

and the $n$th transmitted data vector is

$$
\mathbf{d}_{n}=\left[d_{n, 0}, d_{n, 1}, \ldots, d_{n, N-1}\right]^{T} .
$$

The channel convolution matrix $\tilde{C}_{h}$ is an $N \times N$ circulant matrix using the channel FIR impulse response $\mathbf{h}=\left[h_{0}, h_{1}, \ldots, h_{L}\right]^{T}$ padded with $N-L-1$ zeros as its first column.

Each received data vector $\mathbf{r}_{n}$ is transformed, using FFT, to form the FD vector $\mathbf{R}_{n}$

$$
\mathbf{R}_{n}=\mathbf{H} \circ \mathbf{D}_{n}+\mathbf{V}_{n}
$$

where $\mathbf{D}_{n} \in \mathbb{C}^{N \times 1}$ and $\mathbf{V}_{n} \in \mathbb{C}^{N \times 1}$ are the $N$-point FFT of $\mathbf{d}_{n}$ and $\mathbf{v}_{n}$, respectively. $\mathbf{H} \in \mathbb{C}^{N \times 1}$ is the FD channel impulse response, i.e., the $N$-point FFT of $\mathbf{h}$. The FDE is formulated by $\mathbf{Y}_{n}=\mathbf{W} \circ \mathbf{R}_{n}$, where $\mathbf{W}=\left[W_{0}, W_{1}, \ldots, W_{N-1}\right]^{T}$ is the equalization vector, with $W_{k}(k=0, \ldots, N-1)$ being the weight for each frequency bin. So the FD output error vector is

$$
\mathbf{E}_{n}=\mathbf{D}-\mathbf{W} \circ \mathbf{H} \circ \mathbf{D}_{n}-\mathbf{W} \circ \mathbf{V}_{n}
$$

Then $\mathbf{Y}_{n}$ is inverse transformed via IFFT to form the TD output $\mathbf{y}_{n}$ and detection is done afterward.

\section{B. SM Frequency-Domain Equalization}

The objective here is to find $\mathbf{W}$ according to F-SMF's bounded error-vector criterion. Assume that the transmitted symbols $\left\{d_{n, k}\right\}$ come from a 2-D constellation $\mathcal{C}$, and the equalizer size is $N$. Note that the set of transmit vectors $d$ is $\mathcal{C}^{N}$. With equalizer $\mathbf{W}$, the maximum Euclidean distance between the transmitted vector and equalized output vector for each transmit vector $\mathbf{d}_{n} \in \mathcal{C}^{N}$ is

$$
\begin{aligned}
& Z_{\max }\left(\mathbf{d}_{n}\right) \\
& =\sup _{\mathbf{V} \in \mathcal{V}}\left\|\mathbf{D}_{n}-\mathbf{W} \circ \mathbf{H} \circ \mathbf{D}_{n}-\mathbf{W} \circ \mathbf{V}\right\| \\
& =\sup _{\mathbf{V} \in \mathcal{V}} \sqrt{\sum_{k=0}^{N-1}\left|\left(1-W_{k} H_{k}\right) D_{n, k}-W_{k} V_{k}\right|^{2}}
\end{aligned}
$$

where the set $\mathcal{V}$ consists of all the noise components that are bounded

$$
\mathcal{V} \triangleq\left\{\mathbf{V} \in \mathbb{C}^{N \times 1}:\|\mathbf{V}\|^{2} \leq \gamma_{v}^{2}\right\}
$$

for some noise bound $\gamma_{v}$.

With the error-bound specification $\gamma_{f}$, the objective of SM-FDE is to find $\mathbf{W}$ such that $Z_{\max }(\mathbf{d})$ is upper bounded by $\gamma_{f}$ for any $\mathbf{d} \in \mathcal{C}^{N}$, i.e.,

$$
\sup _{\mathbf{d} \in \mathcal{C}^{N}} Z_{\max }(\mathbf{d}) \leq \gamma_{f}
$$

We define the set of all FDE weight vectors that satisfy (20) as the SM-FDE feasibility set $\mathcal{W}\left(N, \gamma_{f}\right)$

$$
\mathcal{W}\left(N, \gamma_{f}\right) \triangleq \bigcap_{\mathbf{d} \in \mathcal{C}^{N}}\left\{\mathbf{W}: \in \mathbb{C}^{N \times 1}: Z_{\max }(\mathbf{d}) \leq \gamma_{f}\right\}
$$

Note that the SM-FDE feasibility set is a convex set. This follows from the fact that, for each $\mathbf{d} \in \mathcal{C}^{N}, Z_{\max }(\mathbf{d}) \leq \gamma_{f}$ describes the intersection of ellipsoids in the FDE equalization parameter space. Note that the SM-FDE feasibility set could be empty for some particular choice of $N$ and $\gamma_{f}$ given the symbol constellation $\mathcal{C}$, noise set $\mathcal{V}$, and channel $\mathbf{h}$. In general, a larger $\gamma_{f}$ will help ensure that the SM-FDE feasibility set is nonempty. However, a smaller value of $\gamma_{f}$ usually results in better equalization performance. From (18)-(21), one can see that whether or not there exists a feasible SM-FDE solution depends on the channel $\mathbf{h}$, constellation $\mathcal{C}$, noise set $\mathcal{V}$, FFT frame size $N$, and bound $\gamma_{f}$. The following proposition provides sufficient and necessary conditions for the existence of a nonempty SM-FDE feasibility set. For simplicity of discussion, assume that the input symbols and noise are zero-mean independent and identically distributed (i.i.d.) sequences and that the noise and input symbols are uncorrelated.

Proposition 1: Consider a linear FIR channel model and a linear frequency-domain equalizer described in (16)-(21). Let $\gamma_{\bar{d}}$ be the maximum amplitude in the constellation $\mathcal{C}$ and $\mathcal{W}\left(N, \gamma_{f}\right)$ be defined in (21). For each parameter estimate $\mathbf{W}$, the following is true. 
1) Sufficiency: $\mathbf{W}$ is a feasible solution in $\mathcal{W}\left(N, \gamma_{f}\right)$ if

$$
\gamma_{\bar{d}}^{2}\left|1-W_{k_{1}} H_{k_{1}}\right|^{2}+\gamma_{v}^{2}\left|W_{k_{2}}\right|^{2} \leq \frac{\gamma_{f}^{2}}{2 N}
$$

where

$$
\begin{aligned}
& k_{1} \triangleq \arg \max _{k=0, \ldots, N-1}\left|1-W_{k} H_{k}\right| \\
& k_{2} \triangleq \arg \max _{k=0, \ldots, N-1}\left|W_{k}\right| .
\end{aligned}
$$

2) Necessity: If $\mathbf{W}$ is in $\mathcal{W}_{f}(N, \gamma)$, then

$$
\begin{aligned}
\sigma_{d}^{2} \sum_{k=0}^{N-1}\left|1-W_{k} H_{k}\right|^{2}+\sigma_{v}^{2} \sum_{k=0}^{N-1}\left|W_{k}\right|^{2} \\
+2 \sigma_{d} \sigma_{v} \sum_{k=0}^{N-1}\left|W_{k}\right|\left|1-W_{k} H_{k}\right| \leq \gamma_{f}^{2}
\end{aligned}
$$

where $\sigma_{d}$ and $\sigma_{v}$ are the variance of the symbols in the constellation $\mathcal{C}$ and noise, respectively.

Proof: See Appendix I.

Remark 1: Similar conditions have been derived for symbolwise time-domain linear equalizer and decision feedback equalizer in [10] and [25].

Remark 2: From (22), one can see that MMSE-FDE [12], [13] is a feasible SM-FDE solution if the error bound specification $\gamma_{f}$ [as defined in (20)] satisfies

$$
\frac{\gamma_{\bar{d}}^{2}}{\left(\left|H_{k_{3}}\right|^{2} \sigma_{d}^{2} / \sigma_{v}^{2}+1\right)^{2}}+\frac{\gamma_{v}^{2}}{\left(\left|H_{k_{4}}\right|+\frac{\sigma_{v}^{2}}{\sigma_{d}^{2}\left|H_{k_{4}}\right|}\right)^{2}} \leq \frac{\gamma_{f}^{2}}{2 N}
$$

where

$$
\begin{aligned}
& k_{3} \triangleq \arg \min _{k=0, \ldots, N-1}\left|H_{k}\right| \\
& k_{4} \triangleq \arg \min _{k=0, \ldots, N-1}\left|H_{k}\right|+\frac{\sigma_{v}^{2}}{\sigma_{d}^{2}\left|H_{k}\right|} .
\end{aligned}
$$

Thus MMSE-FDE for channel $\mathbf{H}$ belongs to the SM-FDE feasibility set. In particular, MMSE-FDE can ensure that the norm of equalized output squared error is upper bounded by $\gamma_{f}^{2}$ which satisfies (24).

These conditions can help an off-line design of SM-FDE based on criterion (20). With the knowledge of channel $\mathbf{h}$, constellation $\mathcal{C}$, equalizer length $N$ and performance specification $\gamma_{f}$, the design problem is one of finding a parameter estimate that belongs to the feasibility set. The design methodology presented in [25] can be employed here.

In employing SM-FDE for most practical problems (e.g., wireless communications), the feasibility set can not be calculated a priori because the channel $\mathbf{h}$ is not known a priori and might even be time-varying. An adaptive solution is thus required, and the F-SM-NLMS algorithm presented in the previous section can be employed here. To examine the performance of adaptive SM-FDE, consider the same microwave radio channel as in the previous simulation example. An FDE with $N=128$ is considered and the channel impulse response is truncated to 20 most significant coefficients. The

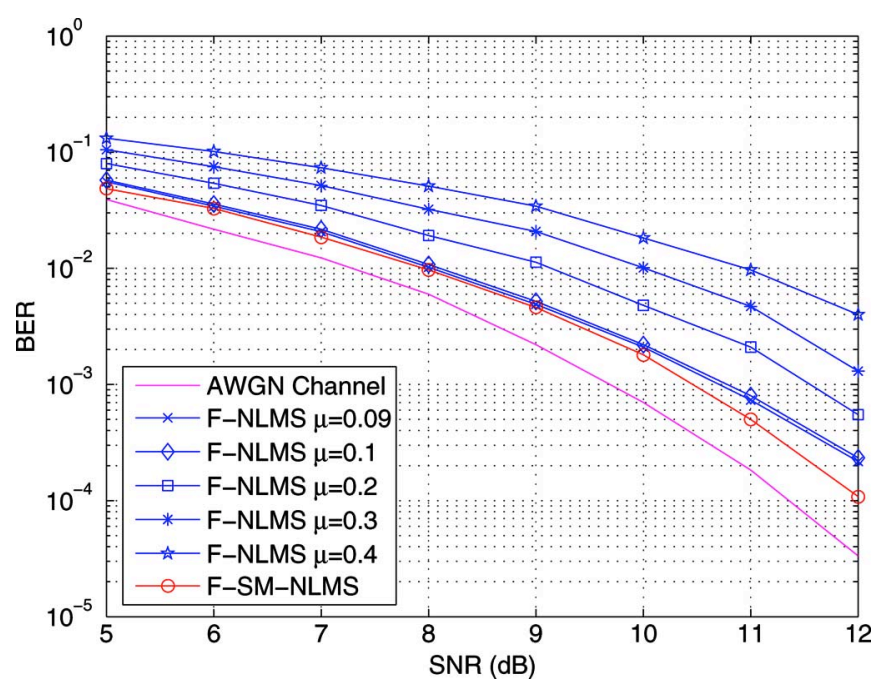

Fig. 4. BER performance with QPSK signaling.

FDE performance using F-SM-NLMS is compared with that of the F-NLMS algorithm implemented with different values of step-size. Fig. 4 shows the steady-state BER for the case of QPSK (Quadrature Phase Shift Keying) signaling. For each simulation, 10000 data vectors, each of which consisting of $N=128$ symbols were transmitted. The bound $\gamma_{k}$ for each bin used in F-SM-NLMS is $\sqrt{5} \sigma_{v}$ (which implies $\gamma_{f}=8 \sqrt{10} \sigma_{v}$ ). In the application of adaptive FDE, $\gamma_{k}$ in F-SM-NLMS should be different for different bins. The reason is that different frequency bins generally have different channel response coefficients. However, in adaptive FDE, we can not specify different $\gamma_{k}$ for each individual bin because $\mathbf{h}$ is not known a priori and can be time-varying. In the next subsection, this problem will be further discussed and we will present an adaptive algorithm to resolve this problem. For comparison, we also show the performance of QPSK signaling in AWGN noise (without ISI). This is equivalent to using a filter matched to the FIR channel. We see that F-SM-NLMS results in a lower BER compared to the F-NLMS, while using only $5 \%$ of data to update parameter estimates. However, as the step-size of F-NLMS is progressively reduced from 0.4 to 0.09 , its performance approaches that of F-SM-NLMS. In practice, the step-size usually varies from 0 to 1 and its choice is often empirical and ad hoc.

\section{An Adaptive Algorithm With Parameter-Dependent Error-Bound Tuning}

One of the critical assumptions of SMF and F-SMF algorithms is the a priori knowledge of an error bound (as in F-SMNLMS). The SM algorithms' performance may depend critically on this specification. In practice, however, it is often not easy to determine the error bound accurately due to insufficient knowledge and/or the time-varying characteristics of the underlying system. Choosing error bounds arbitrarily may be unreliable and has the risk of overbounding (i.e., the error bound is too large) and underbounding (i.e., the error bound is too small); both of which can result in performance degradation. These situations call for alternative SMF algorithms, e.g., those with bound-tuning strategies [26]-[30] that have the ability of 
adjusting the error bound in accordance with the changes in the system.

In this paper, we propose to modify the F-SMF criterion (5) by replacing the fixed $\Gamma^{f}$ with a variable error-bound vector that depends on the filter weights. To begin with, denote the error bound vector as $\Gamma^{f}(\mathbf{W})=\left[\gamma_{0}(\mathbf{W}), \ldots, \gamma_{N-1}(\mathbf{W})\right]^{T}$ to emphasize its dependence on the filter parameter vector. Its value is the true absolute error if $\mathbf{W}$ is a true feasible solution for the filter parameter vector. With the specified error bound function $\Gamma^{f}(\mathbf{W})$, the new criterion is to find $\mathbf{W}$ such that the output error $E_{k}(\mathbf{W})$ is not greater than $\gamma_{k}(\mathbf{W})$ for all possible transmitted symbol vector-received vector pair $(\mathbf{D}, \mathbf{R})$, i.e.,

$$
\begin{array}{r}
\left|E_{k}(\mathbf{W})\right|=\left|\left(1-W_{k} H_{k}\right) D_{k}-W_{k} V_{k}\right| \leq \gamma_{k}(\mathbf{W}) \\
\text { for all } k=0,1, \ldots, N-1 .
\end{array}
$$

To derive a recursive solution based on this new criterion, assume that a sequence of input-pilot data pair $\left(\mathbf{D}_{n}, \mathbf{R}_{n}\right)$ is available for "training." At time $n$, given $\left(\mathbf{D}_{n}, \mathbf{R}_{n}\right)$, the constraint set $\mathcal{H}_{n}$ is defined as the set of all parameters that satisfy the error-bound specification

$$
\begin{array}{r}
\mathcal{H}_{n} \triangleq \bigcap_{k=0}^{N-1}\left\{\mathbf{W} \in \mathbb{C}^{N \times 1}: \mid\left(1-W_{k} H_{k}\right) D_{n, k}\right. \\
\left.-W_{k} V_{n, k} \mid \leq \gamma_{k}(\mathbf{W}) .\right\}
\end{array}
$$

A recursive solution can be derived with a point-wise approach [6]. Given the parameter estimate $\mathbf{W}_{n-1}$ at time $n-1$, and the input-pilot pair $\left(\mathbf{D}_{n}, \mathbf{R}_{n}\right)$, a new estimate $\mathbf{W}_{n}$ is found by $\mathbf{W}_{n}=\arg \min _{\mathbf{W} \in \mathcal{H}_{n}}\left\|\mathbf{W}-\mathbf{W}_{n-1}\right\|^{2}$. This algorithm is outlined here.

1. If $\mathbf{W}_{n-1} \in \mathcal{H}_{n}$, then $\mathbf{W}_{n}=\mathbf{W}_{n-1}$, and no parameter update is needed.

2. If $\mathbf{W}_{n-1} \notin \mathcal{H}_{n}, W_{n}$ is found by

$$
\begin{aligned}
& \mathbf{W}_{n}=\arg \min _{\mathbf{W} \in \mathbb{C}^{N \times 1}}\left\|\mathbf{W}-\mathbf{W}_{n-1}\right\|^{2} \\
& \text { such that }\left|\left(1-W_{k} H_{k}\right) D_{n, k}-W_{k} V_{n, k}\right| \leq \gamma_{k}(\mathbf{W}) \\
& \forall k=0,1, \ldots, N-1 .
\end{aligned}
$$

The algorithm can only be specified according to the specific functional form of $\gamma_{k}(\mathbf{W})$. Assume that the noise distribution has a variance $\sigma_{v}^{2}$. For each bin, with the equalization parameter $W_{k}$, the noise part in the equalization output is $W_{k} V_{k}$ with variance $\left|W_{k}\right|^{2} \sigma_{v}^{2}$. If $W_{k}$ is a feasible equalizer weight, $\sqrt{\alpha\left|W_{k}\right|^{2} \sigma_{v}^{2}}$ can be used to approximate the error bound for some positive constant $\alpha$. So we can set $\gamma_{k}\left(W_{k}\right)=\sqrt{\alpha\left|W_{k}\right|^{2} \sigma_{v}^{2}}$, where $\alpha$ should be greater than 1 . It should be noted that $\gamma_{k}\left(W_{k}\right)$ should be large enough to warrant a non-empty feasibility set, but it should not be too large to slow down convergence. In general, if $\alpha$ is set in the range of [3,9], the performance is satisfactory in AWGN case. Practically, the noise variance may also be unknown. Methods for estimating noise variance can be found in [31] and [32]. As an example, an online estimation of the noise variance and signal-to-noise ratio (SNR) of real processes was presented in [31]. Though it is possible that $\alpha \hat{\sigma}_{v}^{2}<\sigma_{v}^{2}$ (due to the errors in the estimate of $\sigma_{v}^{2}$ ), it does not usually lead to a catastrophe. Furthermore, that probability is usually very small in practice. The recursive solution is summarized as follows (here, we drop the bin index in all variables and the results are equally applicable to all bins).

1) If

$$
\left|E_{n}\right|^{2} \triangleq\left|D_{n}-R_{n} W_{n-1}\right|^{2} \leq \alpha\left|W_{n-1}\right|^{2} \sigma_{v}^{2}
$$

then $W_{n}=W_{n-1}$ and no parameter update is needed.

2) Else, $W_{n}$ is found by

$$
\begin{aligned}
W_{n}=\arg \min _{W \in \mathbb{C}} & \left|W-W_{n-1}\right|^{2} \\
\text { subject to: } & \left|D_{n}-R_{n} W\right|^{2} \leq \alpha|W|^{2} \sigma_{v}^{2} .
\end{aligned}
$$

Define $\beta_{n} \triangleq\left|R_{n}\right|^{2}-\alpha \sigma_{v}^{2}$, constraint (29) may lead to different solutions for $W_{n}$ with different values of $\beta_{n}$. The adaptive algorithm for FDE is summarized as follows (please see Appendix II for derivation).

If

$$
\left|E_{n}\right| \leq \sqrt{\alpha\left|W_{n-1}\right|^{2} \sigma_{v}^{2}}
$$

then

$$
W_{n}=W_{n-1}
$$

else

$$
\begin{aligned}
\lambda_{n}= & \sqrt{\alpha} \sigma_{v}\left|D_{n}\right| \\
\beta_{n}= & \left|R_{n}\right|^{2}-\alpha \sigma_{v}^{2} \\
\xi_{n}= & D_{n} R_{n}^{*}-\beta_{n} W_{n-1} \\
W_{n}= & \left\{\begin{array}{c}
\frac{1}{2}\left(W_{n-1}-W_{n-1}^{*} \frac{\xi_{n}}{\xi_{n}^{*}}+\frac{\xi_{n}}{\left|R_{n}\right|^{2}}\right) \\
\text { if } \beta_{n}=0 \\
W_{n-1}+\left(1-\frac{\lambda_{n}}{\left|\xi_{n}\right|}\right) \frac{\xi_{n}}{\left|\beta_{n}\right|} \\
\text { if } \beta_{n}>0 \\
W_{n-1}+\left(1-\frac{\left|\xi_{n}\right|}{\lambda_{n}}\right) \frac{\lambda_{n} \xi_{n}}{\left|\beta_{n}\right| \cdot\left|\xi_{n}\right|} \\
\text { if } \beta_{n}<0
\end{array}\right.
\end{aligned}
$$

This recursive algorithm derived above is referred to here as SM parameter-dependent error-bound tuning (SM-PET) and it retains the advantages of conventional recursive SMF solutions, e.g., data-dependent step-size and selective update. In addition, the step-size is dependent on the filter parameter vector. The parameter-dependent error-bound specification and step-size make the adaptive algorithm less vulnerable to overbounding and underbounding, thus, providing better convergence and tracking performance.

\section{Performance of Adaptive Algorithms With Variable Bounds}

Simulation experiments were performed to examine the SM-PET algorithm. In all examples presented here, FDE with a 64-point FFT is employed. The channel model used in the simulation is again a microwave radio channel obtained from actual field measurements [20]. A QPSK signaling is assumed. The value of $\alpha$ in defining $\gamma_{k}\left(W_{k}\right)=\sqrt{\alpha\left|W_{k}\right|^{2} \sigma_{v}^{2}}$ is set to be 5. The results of the SM-PET algorithm are compared with those of the F-SM-NLMS algorithm, which uses a fixed error 


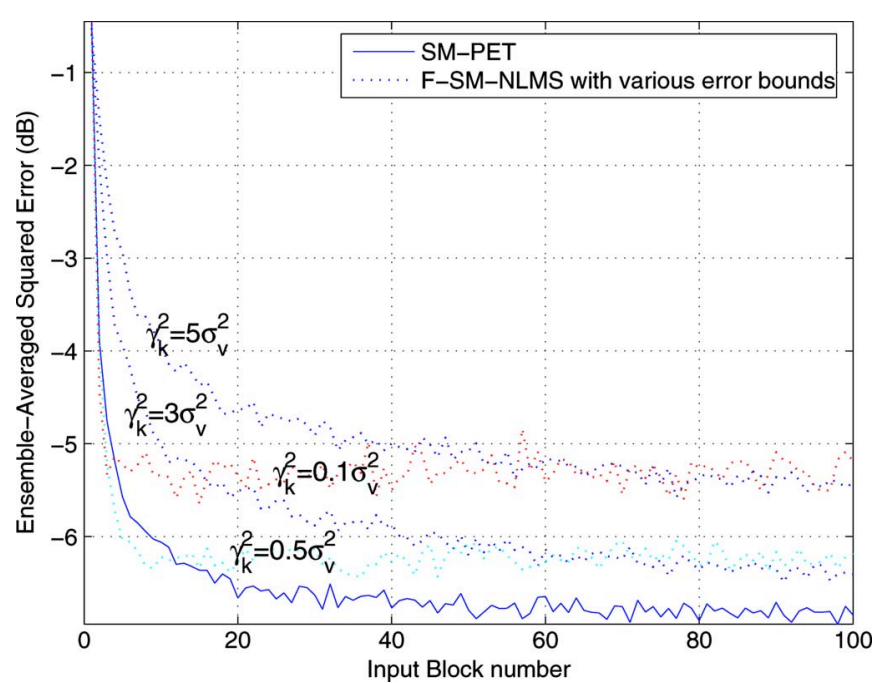

Fig. 5. Convergence performance with Gaussian noise: SM-PET algorithm versus F-SM-NLMS with different error bounds.

bound, and F-NLMS algorithm [18]. For the same reason in the simulation of Fig. 4, F-SM-NLMS algorithm uses the same $\gamma_{k}$ for different bins.

Example 1: Convergence Performance in Gaussian Noise: In this simulation, the input noise is AWGN with zero mean and variance $\sigma_{v}^{2}=0.1$. The convergence performance of SM-PET is compared to that of F-SM-NLMS with various error bounds, as shown in Fig. 5. To illustrate the effect of overbounding/underbounding on performance, we have chosen some extreme values of the error bounds for F-SM-NLMS. As expected, the performance of F-SM-NLMS varies substantially with different (extreme) choices of the error bound. Arbitrarily choosing the error bound with insufficient information could seriously compromise the performance. Simulation results also show that SM-PET enjoys sparse data-dependent updates (like all SMF algorithms) and the update frequency is significantly less than that of F-SM-NLMS. In this example, the updates needed for SM-PET were only $4 \%$. In contrast, the updates in F-SM-NLMS were $46 \%, 32 \%, 3.2 \%$, and $2.5 \%$ for the bounds of $0.1 \sigma_{v}^{2}, 0.5 \sigma_{v}^{2}, 3 \sigma_{v}^{2}$, and $5 \sigma_{v}^{2}$, respectively. The performance comparison between SM-PET algorithm and F-NLMS algorithm is shown in Fig. 6. The SM-PET algorithm is clearly shown to have faster convergence and lower steady-state error than F-NLMS algorithm, which requires a priori setting of the step-size. Comparing results in Figs. 5 and 6, one sees that F-SM-NLMS with $\gamma^{2}=5 \sigma_{v}^{2}$ and F-NLMS with $\mu=0.4$ have the same convergence rate but the F-SM-NLMS has much lower steady-state than F-NLMS in this case.

Example 2: Equalization of a Time-Variant Channel: The channel model used in the previous simulation was set as the initial state of the channel, and the coefficients were then varied randomly. The time variations in the coefficients were introduced by having random Gaussian jumps every 200 data blocks and the variations in the first channel tap are shown in Fig. 7. The curves shown in Fig. 8 are learning curves of SM-PET and F-SM-NLMS with various error bounds. It is clear that SM-PET tracked the channel quite well, while F-SM-NLMS with arbitrarily chosen error bound could not ensure satisfactory equal-

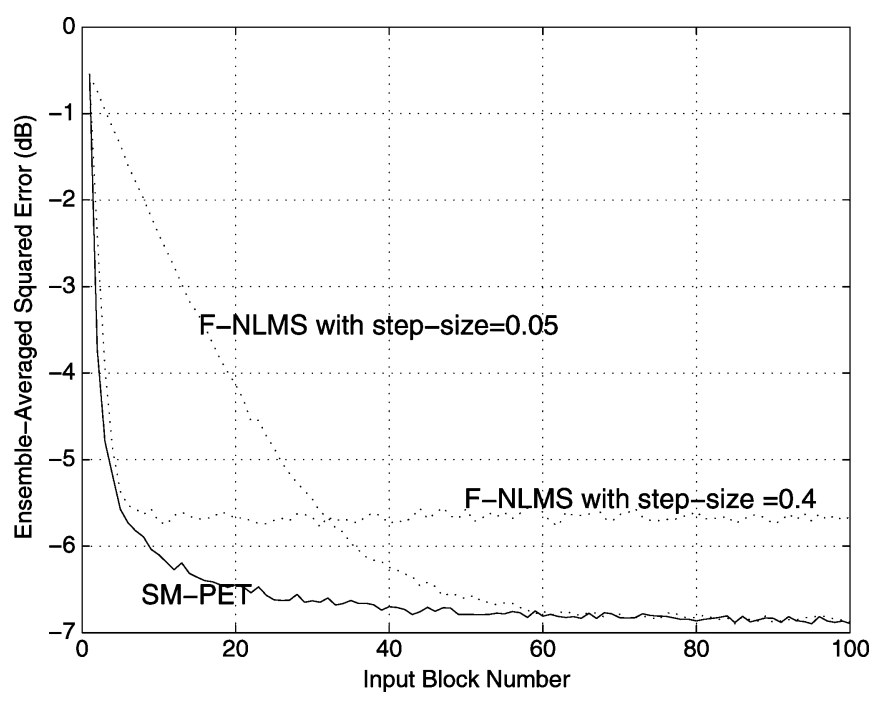

Fig. 6. Convergence performance with Gaussian noise: SM-PET algorithm versus F-NLMS algorithm.

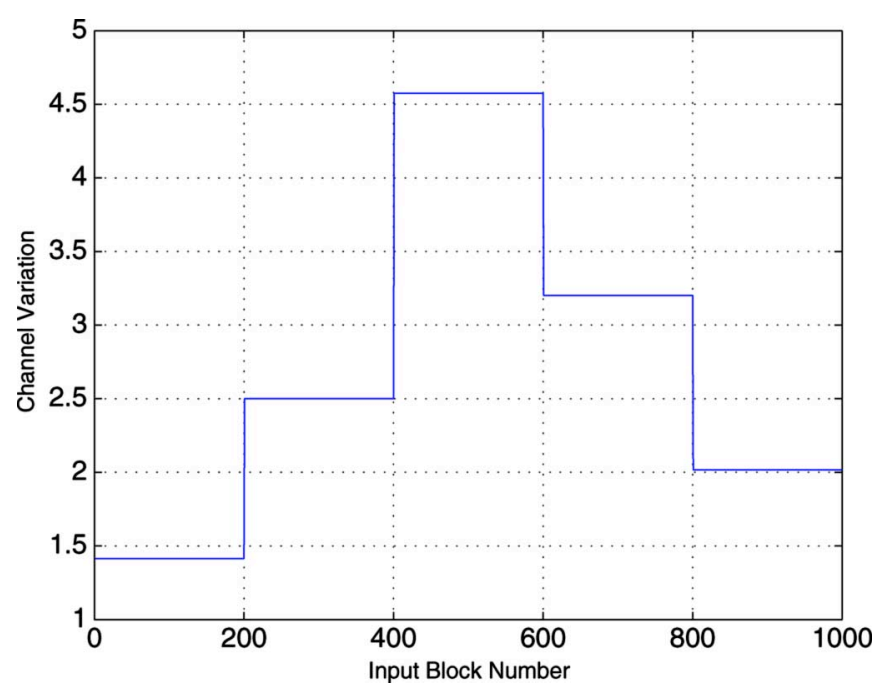

Fig. 7. Time variations of channel.

ization performance. The F-SM-NLMS algorithm with $\gamma^{2}=\sigma_{v}^{2}$ could provide comparable performance to SM-PET in some intervals, but it rendered inferior performance in other intervals. In essence, for the equalization of time-varying channels, a fixed value of $\gamma_{k}$ (as employed in F-SM-NLMS) can not guarantee robust equalization performance. Other experiments of SM-PET in non-Gaussian noise showed similar results.

\section{UPDATOR-SHARING SCHEME FOR BROADBAND WIRELESS COMMUNICATIONS}

Due to the unique data-dependent selective update feature of all SM adaptive algorithms, the adaptation process is essentially decomposed into two steps: information evaluation (which decides whether or not an update of parameter estimate is needed) followed by update calculation, if it is needed. When updates are infrequent, one can conceivably design shared updators among parallel adaptive filters with SM algorithms, thereby reducing hardware requirement. This idea was first presented in [10] and referred to as U-SHAPE there. It will be shown in this sec- 


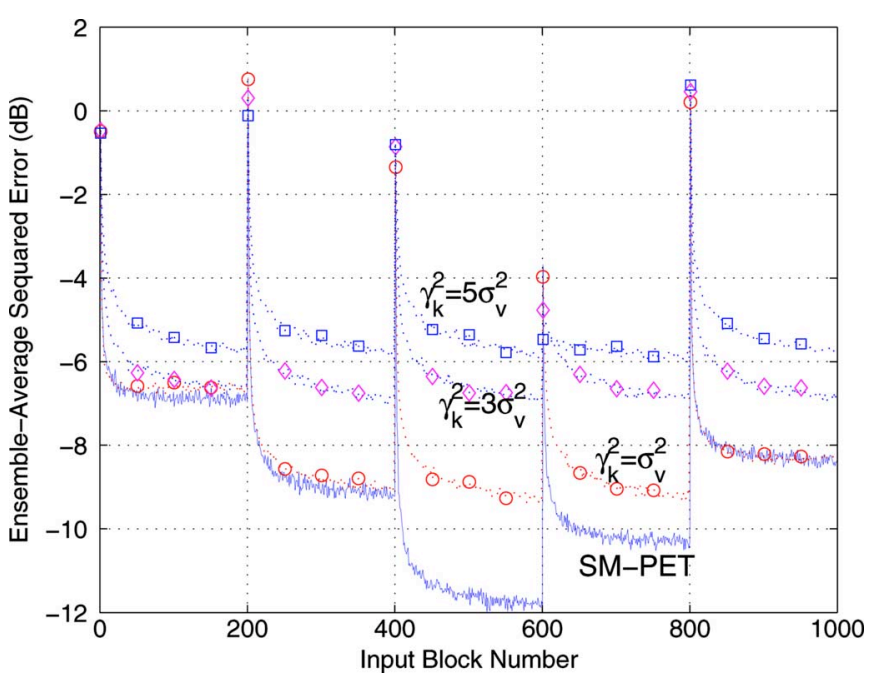

Fig. 8. Tracking of time-variant channel: SM-PET algorithm (solid line without markers) versus F-SM-NLMS with different error bounds (dotted lines with markers).

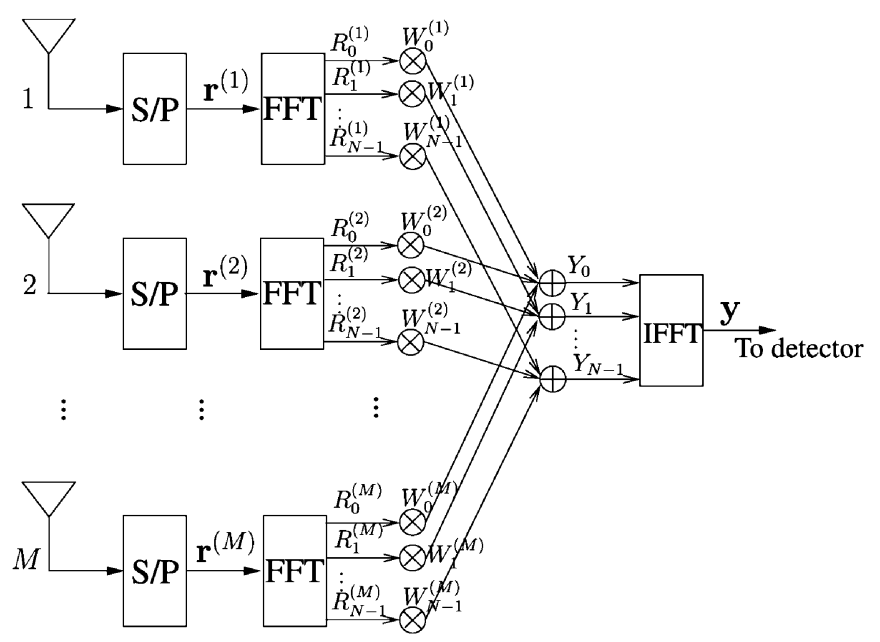

Fig. 9. Frequency-domain diversity combining and equalization.

tion, with the idea of U-SHAPE, that employing F-SMF in frequency-domain diversity combining and equalization can provide a very appealing receiver structure with low hardware complexity for broadband wireless communications.

\section{A. Frequency-Domain Diversity Combining and Equalization}

Frequency-domain diversity combining and equalization, as shown in Fig. 9, is considered a robust and efficient receiver solution for very dispersive channels like those in broadband wireless communications [13], [33]. Consider a single-carrier block-wise transmission with $\mathrm{CP}$ as described earlier. Assume that there are $M$ receive antennas. At the $m$ th receive branch, each data vector $\mathbf{r}^{(m)} \in \mathbb{C}^{N \times 1}$ is transformed through FFT after the $\mathrm{CP}$ is removed. Then the combination and equalization are conducted in frequency domain. The signals at the same frequency bin from different diversity branches are first combined and the resulting combined signal is then equalized. The output at the $k$ th bin is $Y_{k}=\mathbf{W}_{k}^{T} \mathbf{R}_{k}(0 \leq k \leq N-1)$, where

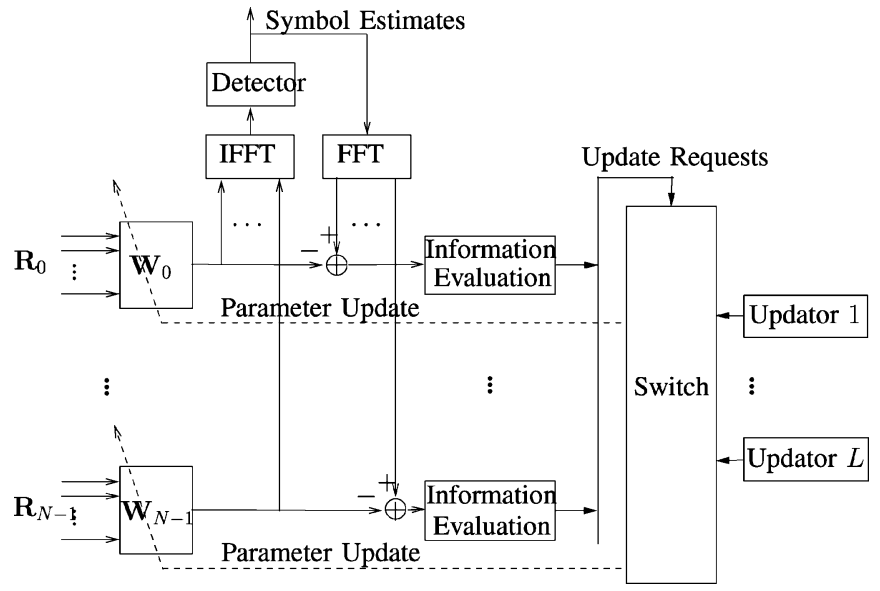

Fig. 10. Structure of updator-sharing scheme.

$\mathbf{W}_{k}=\left[W_{k}^{(1)}, \ldots, W_{k}^{(M)}\right]^{T}$ is the combination and equalization parameter vector and $\mathbf{R}_{k}$ is $\left[R_{k}^{(1)}, \ldots, R_{k}^{(M)}\right]^{T}$. Then the output is transformed back into time domain and the decision is made. This receiver structure can provide the multipath diversity gain as well as multiple antenna diversity gain, thus yields excellent performance in very dispersive radio channels. It has been shown that an $M$-branch receiver with maximal ratio combining (MRC) and LMMSE equalization performs better than an $(M-1)$-branch maximum likelihood receiver in highly dispersive fading radio channels with AWGN noise [13].

If the input statistics and the channel information of each subchannel are known perfectly, the optimum combining and equalization weight $\mathbf{W}_{k}$ can be obtained from MRC and LMMSE [13], [33]. However, such information is often unknown in practice, thus adaptive algorithms would be preferred. The F-SMNLMS and SM-PET algorithm can be employed here for adaptive combination and equalization.

\section{B. Updator-Sharing Scheme}

With the receiver structure presented previously, each bin has an adaptive filtering subsystem with $M$ inputs and one output, and there are $N$ parallel such subsystems with the same filter and update structure. Employing F-SMF in this receiver structure allows for sharing updators among those $N$ subsystems to reduce the hardware requirement [10]. Decision-directed scheme is employed in the proposed receiver. The proposed sharing scheme is illustrated in Fig. 10 and described briefly as follows.

- For each bin $k$, if the error $\left|E_{n, k}\right|$ does not satisfy (30) [or (10)] through the information evaluation, an update request is sent to the updator switch control.

- If the number of update requests $N_{r} \leq L$, all requests are responded to, i.e., the filters which send update requests are all updated accordingly. If $N_{r}>L,\left(N_{r}-L\right)$ requests will have to be declined.

To design such an updator-sharing scheme, the following issues must be addressed [10].

1) The Number of Updators L: The goal is to have the minimum number of updators without compromising the overall system's performance. Determination of $L$ would depend on the 
update request probability $p_{k}$ and the targeted rejection probability $\rho_{o, k}$ for each bin. The rejection probability for each bin is defined as

$$
\rho_{k}=\operatorname{Prob}\{\text { denial } \mid \text { request }\} .
$$

Denote $\bar{L}$ as the minimum number of updators to satisfy $\rho_{k} \leq$ $\rho_{o, k}$ for all $k$. The value of $\bar{L}$ can be obtained from ([10, Th. 2 and Th. 3]) by searching in the set of possible values $L \in$ $\{1,2, \ldots, N\}$ off-line. A higher rejection probability implies that fewer update requests can be fulfilled and performance may be compromised. According to our simulation experience, the BER performance is usually not compromised much if $\rho_{o, k} \leq$ 0.1 . Further, since all the bins are from the same user, it makes sense to use the same value of $\rho_{o, k}$ for all bins.

2) The Update Request Probability $p_{k}$ : To design $L$, one needs to specify the update request probability as a system parameter. There are two relevant issues here. The first is that $p_{k}$ is time-varying in practice. Usually, $p_{k}$ is larger at the beginning of equalization and decreases to a smaller steady-state value as equalization proceeds. If we set the request probability according to $p_{\text {init }}$, the value for the training period, it may be too conservative and will require an unnecessarily large number of updators. On the other hand, if we set the request probability according to the steady-state value $p_{\mathrm{ss}}$, one risks slowing down convergence. Our experience suggests that using $p_{\mathrm{ss}}$ will be satisfactory, because the SM-PET and F-SM-NLMS algorithms usually reach the steady-state very quickly. Thus, the initial training period is insignificant in the time frame of interest. While choosing a smaller update probability may slow down convergence, this is insignificant according to our simulation experience.

The second issue is that different SNRs and channel realizations would result in different $p_{\mathrm{ss}}$. We propose to specify $L$ in accordance with the smallest acceptable SNR value.

3) Updator Allocation Scheme: As aforementioned, if $N_{r}>$ $L$, only $L$ update requests are fulfilled and a prioritizing allocation scheme is required. We propose a prioritization scheme to allocate the updators. To begin with, define a priority index $\Delta_{n, k}$ for each update request as

$$
\Delta_{n, k} \triangleq 1-\frac{\gamma_{k}\left(\mathbf{W}_{n-1, k}\right)}{\left|E_{n, k}\right|}
$$

if the SM-PET algorithm is employed here, or

$$
\Delta_{n, k} \triangleq 1-\frac{\gamma_{k}}{\left|E_{n, k}\right|}
$$

if F-SM-NLMS algorithm is employed. Whenever an update is needed, the update request is sent along with $\Delta_{n, k}$ to the switch control. If $N_{r}>L$, the updators are allocated based on the values of $\Delta_{n, k}$. In particular, the updators are allocated to those filters with the $L$ highest values of $\Delta_{n, k}$. The updator-sharing scheme is summarized as follows.

\section{Design Procedure:}

- Determine the SNR region of interest and the channel statistics; evaluate the average $p_{\text {init }}$ and $p_{\mathrm{ss}}$ among all possible channel realizations according to the chosen channel statistical model for the lowest SNR value.
- Choose $p_{k}$ from $\left[p_{\mathrm{ss}}, p_{\text {init }}\right]$ and determine the targeted rejection probability $\rho_{o, k}$.

- Calculate $L$ by searching in the set of $\{1,2, \ldots, N\}$ based on ([10, Th. 2 and Th. 3]).

2. Operation:

- For each bin $k$, if the error $\left|E_{n, k}\right|$ does not satisfy (32) through the information evaluation, an update request is sent along with the priority value $\Delta_{n, k}$ to the updator switch control.

- If the number of update requests $N_{r} \leq L$, all requests are fulfilled, i.e., all the filters which send update request are updated accordingly.

- If $N_{r}>L$, the prioritization scheme (based on $\Delta_{n, k}$ ) is employed to allocate the updators to those $L$ filters with the highest values of $\Delta_{n, k}$ and the remaining $N_{r}-L$ filters are declined service.

\section{Performance Evaluation}

This section examines the effect of sharing updator on the overall system performance. The performance measures considered here are the average equalization convergence rate, average bit error rate (BER) and outage probability. These performance measures are compared for the sharing and the non-sharing (full $N$ updators) schemes.

In the simulations, the channel model is the "SUI-5" model for broadband wireless channels with multipath fading, which is one of the six channel models adopted by IEEE 802.16 for evaluating broadband wireless systems in $2-11 \mathrm{GHz}$ bands [34]. "SUI-5" is a high delay model associated with the use of omnidirectional antennas in suburban hilly environments. The channel has a maximum delay spread of $10 \mu \mathrm{s}$, and an rms delay spread of $3.05 \mu \mathrm{s}$. It has three echoes, at 0,4 , and $10 \mu \mathrm{s}$, modeled as independent complex Gaussian random variables with the relative power of $0,-5,-10 \mathrm{~dB}$, respectively. An $M=2$-branch receiver system was used with FFT length $N=64$. Modulation scheme was QPSK with symbol duration of $1 \mu \mathrm{s}$. So the channel impulse response model is an FIR with 11 taps. In this set of experiments, we employed the SM-PET algorithm and implemented it with and without updator sharing. The training block number was $N_{t}=5$. The SNR of interest ranged from 5 to $15 \mathrm{~dB}$.

First, by averaging over all channel realizations, the average update request probabilities were found to be 0.09 for $\mathrm{SNR}=$ $5 \mathrm{~dB}, 0.07$ for $10 \mathrm{~dB}$, and 0.065 for $15 \mathrm{~dB}$. So we used 0.09 as the system parameter to define the updator number $L$. With the rejection probability set to be $\rho_{0}=0.1$, the minimum updator number was 7 .

The average convergence performance of sharing 7 updators is shown along with that of the non-sharing case (64 updators) in Fig. 11. The average SNR at each diversity branch is $5 \mathrm{~dB}$. The results are obtained by averaging the convergence curves of 20000 random channel realizations. It may be observed that both cases have almost the same steady-state MSE level, while the initial learning curves are slightly different. The reasons may be as follows.

1) The rejection ratio is small, so most of the update requests have been satisfied. 


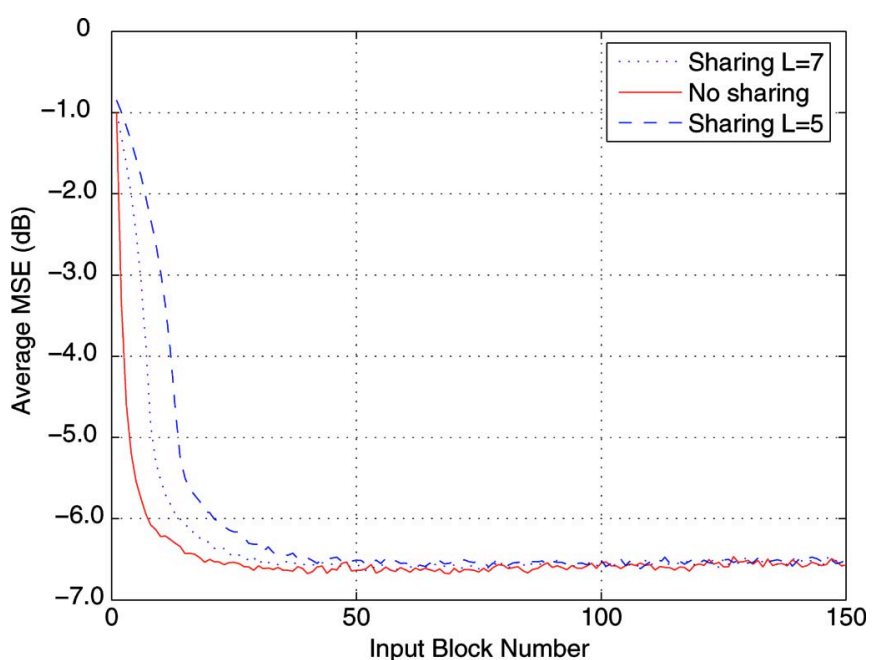

Fig. 11. Learning curves comparison of the sharing case and the non-sharing case.

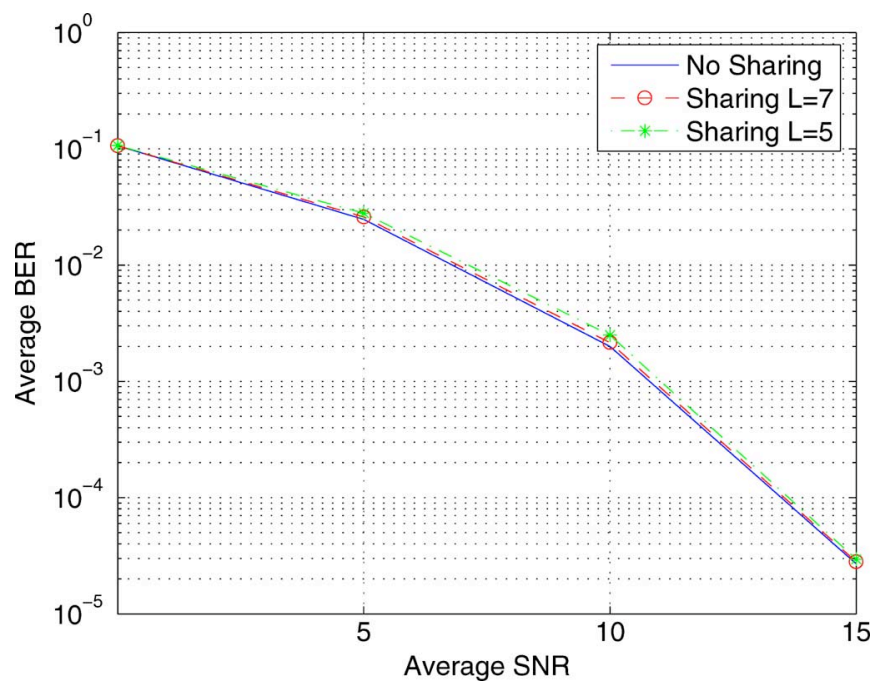

Fig. 12. Average BER comparison of the sharing case and the non-sharing case for QPSK.

2) More importantly, a priority scheme is employed to allocate the updators. So those bins whose update requests are declined usually have smaller equalization errors. Our experience shows that even when some update requests are declined, it does not result in significant performance degradation.

In the training period, the sharing scheme has a larger average MSE than the non-sharing scheme. However, it is observed that almost the same number of blocks are required by both schemes to reach a steady state. To achieve MSE level smaller than $-6 \mathrm{~dB}$, the sharing case with $L=7$ only needs about 5 more data blocks than the non-sharing case. Here those bins with larger errors have higher priority index and larger probability of success in obtaining an update of their filter weights. This results in a uniform convergence over all bins.

Fig. 12 shows the average BER performance comparison between the non-sharing case and the sharing case with $L=7$. Compared with the non-sharing case, the sharing case yields almost the same BER performance. Specifically, to achieve the

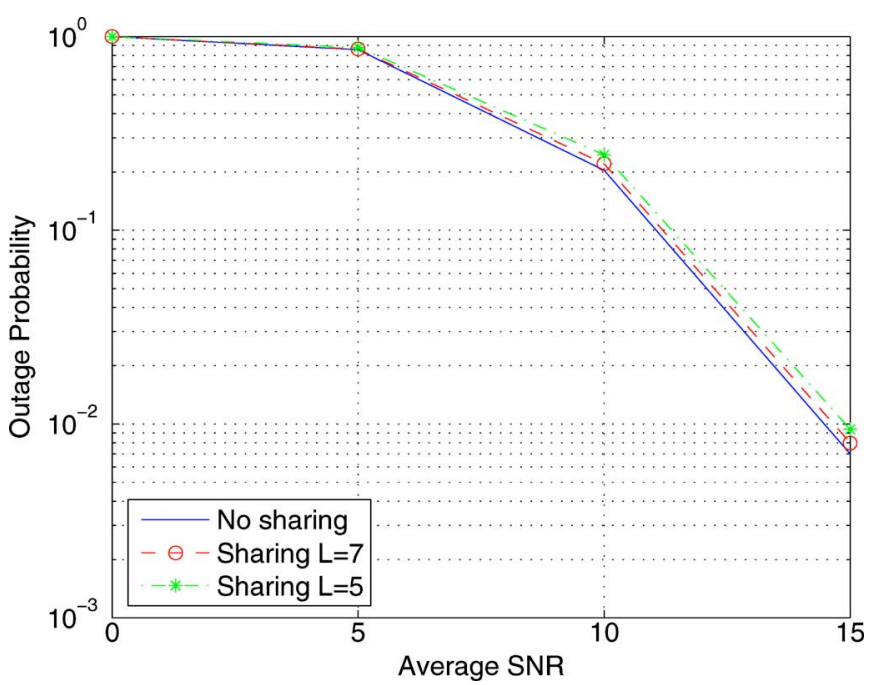

Fig. 13. Outage probability comparison of the sharing case and the non-sharing case for QPSK.

same BER of $10^{-4}$, the sharing case with $L=7$ needs only about $0.1 \mathrm{~dB}$ more than the non-sharing case. Outage probability is another performance measure of wireless communication systems over fading channels [35]. It is defined as the probability that the instantaneous error rate exceeds some threshold value. Fig. 13 shows the outage probability performance comparison between the non-sharing case and the sharing case with $L=7$, where the threshold value is $10^{-4}$. To achieve the same outage probability of 0.01 , the sharing case needs only about $0.2 \mathrm{~dB}$ more than the non-sharing case. This is quite appealing: reducing the hardware complexity drastically ( $L=7$ versus the full $N=64$ updating processors) with very little performance degradation.

\section{Discussion on Robustness With Respect to the Variation of $p_{k}$}

As discussed previously, one of the key parameters in the design of the updator-sharing scheme is the update request probability $p_{k}$. In practice, model deviation (e.g., SNR variation and different channel characteristics) may lead to variations of $p_{k}$, which can affect the performance of the updator-sharing scheme. Usually, a modest decrease in $p_{k}$ would not result in performance degradation. However, an increase in $p_{k}$ would likely result in the violation of $\rho_{k} \leq \rho_{o}$. For a given $L$, a larger value of $p_{k}$ would slow down the convergence and compromise the steady-state MSE performance (and thus compromise the BER performance). The performance only degrades gradually with the increase of $p_{k}$ and the proposed scheme does not lead to a catastrophe with modest variation in $p_{k}$.

The robustness was examined by simulation through either decreasing $L$ or increasing update request probability $p_{k}$. The effect of choosing a smaller $L$ on the convergence performance is also shown in Fig. 11, where $L=5$ was chosen while it is known that the minimum should be 7 . It is seen that the convergence time to $-6 \mathrm{~dB}$ slowed down by about 10 blocks, compared to 5 for $L=7$, for the non-sharing case. However the algorithm still converges to almost the same MSE level. It should be pointed out that for applications where delay is not critical, 
it is possible then to use a smaller $L$ without any significant loss in performance. To demonstrate the robustness of the proposed scheme, Figs. 12 and 13 also include the average BER and outage probability performance for the updator sharing case with $L=5\left(N_{t}=10\right)$ and SNR $<5 \mathrm{~dB}$. We can see that the performance of the sharing case with $L=5$ is very close to the non-sharing case. For SNR smaller than $5 \mathrm{~dB}$, the sharing case (with $L=7$ or $L=5$ ) has comparable performance as compared with the non-sharing case. In essence, the sharing scheme is fairly robust with respect to the system model violation and it can be compensated easily by a few more training blocks.

\section{CONCLUSION}

This paper presented a formulation for frequency-domain set-membership filtering (F-SMF), and an adaptive F-SMF algorithm with an application to frequency-domain equalization. We also derived a novel F-SMF algorithm with parameter-dependent error bounds which are more suitable for adaptive frequency-domain equalization. Results showed that the proposed algorithm has better convergence performance than those MSE-based algorithms. It also outperforms the conventional SMF algorithms, and has less risk of overbounding and underbounding. Finally, an updator-sharing scheme was presented for frequency-domain diversity combining and equalization with reduced hardware complexity requirement.

\section{APPENDIX I}

PROOF OF PROPOSITION 1

Proof: The maximum output error of $\mathbf{d},(18)$, is equal to

$$
\begin{aligned}
Z_{\max }^{2}(\mathbf{d})= & \sup _{\mathbf{V} \in \mathcal{V}} \sum_{k=0}^{N-1}\left\{\left|1-W_{k} H_{k}\right|^{2}\left|D_{k}\right|^{2}\right. \\
& \left.+\left|W_{k}\right|^{2}\left|V_{k}\right|^{2}+2\left|1-W_{k} H_{k}\right|\left|W_{k} D_{k} V_{k}\right|\right\}
\end{aligned}
$$

and is achieved when $\angle\left(V_{k} W_{k}\right)=\pi+\angle\left(\left(1-W_{k} H_{k}\right) D_{k}\right)$ for $k=0, \ldots, N-1$. An upper bound on $Z_{\max }^{2}(\mathbf{d})$ is

$$
Z_{\max }^{2}(\mathbf{d}) \leq 2 \sum_{k=0}^{N-1}\left\{\left|1-W_{k} H_{k}\right|^{2}\left|D_{k}\right|^{2}+\left|W_{k}\right|^{2}\left|V_{k}\right|^{2}\right\}
$$

We know

$$
\sum_{k=0}^{N-1}\left|V_{k}\right|^{2} \leq N \gamma_{v}^{2}
$$

So we have

$$
Z_{\max }^{2}(\mathbf{d}) \leq 2 \sum_{k=0}^{N-1}\left\{\left|1-W_{k} H_{k}\right|^{2}\left|D_{k}\right|^{2}\right\}+2 N\left|W_{k_{2}}\right|^{2} \gamma_{v}^{2}
$$

where

$$
k_{2} \triangleq \arg \max _{k=0, \ldots, N-1}\left|W_{k}\right|
$$

The maximum amplitude in the constellation $\mathcal{C}$ is $\gamma_{\bar{d}}$, so we have

$$
\sum_{k=0}^{N-1}\left|D_{k}\right|^{2} \leq N \gamma \frac{2}{d}
$$

Thus an upper bound of $Z_{\max }^{2}(\mathbf{d})$ is given by

$$
\sup _{\mathbf{d} \in \mathcal{C}^{N}} Z_{\max }^{2}(\mathbf{d}) \leq 2 N\left(\left|1-W_{k_{1}} H_{k_{1}}\right|^{2} \gamma_{\frac{d}{d}}^{2}\right)+2 N\left|W_{k_{2}}\right|^{2} \gamma_{v}^{2}
$$

where

$$
k_{1} \triangleq \arg \max _{k=0, \ldots, N-1}\left|1-W_{k} H_{k}\right|
$$

Then the sufficient condition follows.

For a necessary condition, let's consider the variance of frequency-domain symbol:

$$
\sigma_{D}^{2}=\sigma_{d}^{2} \quad \sigma_{V}^{2}=\sigma_{v}^{2}
$$

So we have

$$
\begin{aligned}
& \sup _{\mathbf{d} \in \mathcal{C}^{N}} Z_{\max }^{2}(\mathbf{d}) \geq \sigma_{d}^{2} \sum_{k=0}^{N-1}\left|1-W_{k} H_{k}\right|^{2} \\
& \quad+\sigma_{v}^{2} \sum_{k=0}^{N-1}\left|W_{k}\right|^{2}+2 \sigma_{d} \sigma_{v} \sum_{k=0}^{N-1}\left|W_{k}\right|\left|1-W_{k} H_{k}\right|
\end{aligned}
$$

and then the necessary condition follows.

\section{APPENDIX II}

DERIVATION OF ADAPTIVE ALGORITHM WITH PARAMETER-DEPENDENT ERROR-BOUND TUNING

Proof: With the error bound function definition $\gamma(W)=$ $\sqrt{\alpha|W|^{2} \sigma_{v}^{2}}$, the recursive solution is found as follows:

1) If

$$
\left|E_{n}\right|^{2}=\left|D_{n}-R_{n} W_{n-1}\right|^{2} \leq \alpha\left|W_{n-1}\right|^{2} \sigma_{v}^{2}
$$

then $W_{n}=W_{n-1}$ and no parameter update is needed.

2) Else, $W_{n}$ is found by

$$
\begin{aligned}
W=\arg \min _{W \in \mathbb{C}} & \left|W-W_{n-1}\right|^{2} \\
\text { subject to: } & \left|D_{n}-R_{n} W\right|^{2} \leq \alpha|W|^{2} \sigma_{v}^{2} .
\end{aligned}
$$

Define $\beta_{n} \triangleq\left|R_{n}\right|^{2}-\alpha \sigma_{v}^{2}$. Based on the value of $\beta_{n}$, constraint (48) may lead to different solutions for $W_{n}$.

1) If $\beta_{n}=0$, constraint (48) can be rewritten as

$$
D_{n} R_{n}^{*} W^{*}+D_{n}^{*} R_{n} W \geq\left|D_{n}\right|^{2} .
$$

The solution for $W_{n}$ is

$$
W_{n}=\frac{1}{2}\left(W_{n-1}-W_{n-1}^{*} \frac{D_{n} R_{n}^{*}}{D_{n}^{*} R_{n}}\right)+\frac{D_{n}}{2 R_{n}} .
$$


2) If $\beta_{n}>0$, constraint (48) can be rewritten as

$$
\left|D_{n} R_{n}^{*}-\beta_{n} W\right|^{2} \leq \alpha \sigma_{v}^{2}\left|D_{n}\right|^{2}
$$

The solution for $W_{k}$ is given by

$$
W_{n}=W_{n-1}+\mu_{n} \frac{\xi_{n}}{\left|\beta_{n}\right|}
$$

where $\xi_{n}=D_{n} R_{n}^{*}-\beta_{n} W_{n-1}$ and $\mu_{n}=1-\left(\sqrt{\alpha} \sigma_{v}\left|D_{n}\right|\right) /$ $\left(\left|\xi_{n}\right|\right)$.

3) If $\beta_{n}<0$, constraint (48) can be rewritten as

$$
\left|D_{n} R_{n}^{*}-\beta_{n} W\right|^{2} \geq \alpha \sigma_{v}^{2}\left|D_{n}\right|^{2} .
$$

The solution for $W_{n}$ is given by

$$
W_{n}=W_{n-1}+\mu_{n}^{\prime} \frac{\sqrt{\alpha} \sigma_{v}\left|D_{n}\right| \xi_{n}}{\left|\beta \xi_{n}\right|}
$$

where $\xi_{n}=D_{n} R_{n}^{*}-\beta_{n} W_{n-1}$ and $\mu_{n}^{\prime}=1-$ $\left(\left|\xi_{n}\right| / \sqrt{\alpha} \sigma_{v}\left|D_{n}\right|\right)$.

Then define $\lambda_{n} \triangleq \sqrt{\alpha} \sigma_{v}\left|D_{n}\right|$ and after some derivation, the result follows.

\section{REFERENCES}

[1] S. Dasgupta and Y. F. Huang, "Asymptotically convergent modified recursive least-squares with data-dependent updating and forgetting factor for systems with bounded noise," IEEE Trans. Inf. Theory, vol. IT-33, no. 3, pp. 383-392, May 1987.

[2] J. R. Deller, Jr., M. Nayeri, and M. S. Liu, "Unifying the landmark developments of optimal bounding ellipsoid identification," Int. J. Adapt. Contr. Signal Process., vol. 8, pp. 48-63, Jan.-Feb. 1994.

[3] E. Walter and H. Piet-Lahanier, "Estimation of parameter bounds from bounded-error data: A survey," Math. Comput. Simul., vol. 32, pp. 449-465, Dec. 1990.

[4] P. S. Diniz and S. Werner, "Set-membership binormalized data Re-using LMS algorithms," IEEE Trans. Signal Process., vol. 51, no. 1, pp. 124-134, Jan. 2003.

[5] S. Werner and P. S. R. Diniz, "Set-membership affine projection algorithm,” IEEE Signal Process. Lett., vol. 8, no. 8, pp. 231-235, Aug. 2001.

[6] S. Gollamudi, S. Nagaraj, S. Kapoor, and Y. F. Huang, "Set-membership filtering and a set-membership normalized LMS algorithm with an adaptive step size," IEEE Signal Process. Lett., vol. 5, no. 5, pp. 111-114, May 1998

[7] M. Milanese, J. Norton, and E. Walter, Bounding Approaches to System Identification. London, U.K.: Plenum, 1996.

[8] L. Guo, A. Ekpenyong, and Y. F. Huang, "Frequency-domain adaptive filtering-A set-membership approach," in Proc. 37th Asilomar Conf. Signals, Syst. Comp., Pacific Grove, CA, 2003, pp. 2073-2077.

[9] D. Joachim and J. R. Deller, Jr., "Short signal classification using setmembership identification: Application to speech labeling," in Proc. 43rd Midwest Symp. Circuits Syst., East Lansing, MI, Aug. 2000, pp. 588-591.

[10] S. Gollamudi, S. Kapoor, S. Nagaraj, and Y. F. Huang, "Set-membership adaptive equalization and an updator-shared implementation for multiple channel communication systems," IEEE Trans. Signal Process., vol. 46, no. 9, pp. 2372-2385, Sept. 1998.

[11] S. Gollamudi and Y. F. Huang, "Iterative nonlinear MMSE multiuser detection," in Proc. IEEE Int. Conf. Acoustics, Speech, Signal Processing (ICASSP), Mar. 15-19, 1999, vol. 5, pp. 2595-2598.
[12] D. Falconer, S. Ariyavisitakul, A. Benyamin-Seeyar, and B. Eidson, "Frequency domain equalization for single-carrier broadband wireless systems," IEEE Commun. Mag., vol. 40, no. 4, pp. 58-66, Apr. 2002.

[13] M. V. Clark, "Adaptive frequency-domain equalization and diversity combining for broadband wireless communications," IEEE J. Sel. Areas Commun., vol. 16, no. 8, pp. 1385-1395, Oct. 1998.

[14] Y. Bendel, D. Burshtein, O. Shalvi, and E. Weinstein, "Delayless frequency domain acoustic echo cancellation," IEEE Trans. Speech Audio Process., vol. 9, no. 5, pp. 589-599, Jul. 2001.

[15] R. M. M. Derkx, G. P. M. Egelmeers, and P. C. W. Sommen, "New constraining method for partitioned block frequency-domain adaptive filters," IEEE Trans. Signal Process., vol. 50, no. 9, pp. 2177-2186, Sep. 2002.

[16] K. Eneman and M. Monnen, "Iterated partitioned block frequency-domain adaptive filtering for acoustic echo cancellation," IEEE Trans. Speech Audio Process., vol. 11, no. 2, pp. 143-158, Mar. 2003.

[17] S. Haykin, Aadaptive Filter Theory, 4th ed. Englewood Cliffs, NJ: Prentice-Hall, 2001.

[18] P. A. Dmochowski and P. J. Mclane, "Frequency domain equalization for high data rate multipath channels," in Proc. IEEE PACRIM, 2001, pp. 534-537.

[19] S. Pushparajah and J. Chambers, "Frequency domain set membership fast normalised Ism adaptive algorithm," Electron. Lett., vol. 35, pp. 865-867, May 1999.

[20] Microwave Channel Database, [Online]. Available: http://spib.rice. edu/spib/microwave.html, Rice Univ.

[21] A. Czylwik, "Comparison between adaptive OFDM and single carrier modulation with frequency domain equalization," in Proc. Vehicular Technology Conf. (VTC), Phoenix, AZ, Mar. 1997, vol. 2, pp. 867-869.

[22] Z. Wang, X. Ma, and G. B. Giannakis, "OFDM or single-carrier block transmission," IEEE Trans. Commun., vol. 52, no. 3, pp. 380-394, Mar. 2004.

[23] T. Pollet, M. Van Bladel, and M. Moeneclaey, "BER sensitivity of OFDM systems to carrier frequency offset and wiener phase noise," IEEE Trans. Commun., vol. 43, no. 2/3/4, pp. 192-193, Feb./Mar./Apr. 1995.

[24] D. Falconer, "Frequency domain equalization for single-carrier broadband wireless systems," Feb. 2002, White Paper, IEEE 802.16.

[25] S. Nagaraj, S. Gollamudi, S. Kapoor, and Y. F. Huang, "BEACON: An adaptive set-membership filtering technique with sparse updates," IEEE Trans. Signal Process., vol. 47, no. 11, pp. 2928-2941, Nov. 1999.

[26] D. Maksarov and J. P. Norton, "Tuning of noise bounds in state bounding," in Proc. Symp. Modelling, Analysis and Simulation (CESA'96), MACS Multiconf., Lille, France, Jul. 9-12, 1996, vol. 2, pp. 837-842.

[27] _ "Tuning of noise bounds in parameter set estimation," in Proc. Int. Conf. Identification in Eng. Syst., Swansea, U.K., Mar. 27-29, 1996, pp. 584-593.

[28] T. M. Lin, M. Nayeriand, and J. R. Deller, Jr., "Consistently convergent OBE algorithm with automatic selection of error bounds," Int. J. Adapt. Contr. Signal Process., vol. 12, pp. 302-324, Jun. 1998.

[29] D. Joachim, J. R. Deller, Jr., and M. Nayeri, "Practical considerations in the use of a new OBE algorithm that blindly estimates error bounds," in Proc. 40th Midwest Symp. Circuits Syst., Sacramento, CA, Aug. 1997, vol. 2, pp. 762-765.

[30] S. Gazor and K. Shahtalebi, "A new NLMS algorithm for slow noise magnitude variation," IEEE Signal Process. Lett., vol. 9, no. 11, pp. 348-351, Nov. 2002.

[31] S. Niu and D. Fisher, "Simutaneous estimation of process parameters, noise variance, and signal-to-noise ratio," IEEE Trans. Signal Process., vol. 43, no. 7, pp. 1725-1728, Jul. 1995.

[32] K. Paliwal, "Estimation of noise variance from the noisy AR signal and its application in speech enhancement," IEEE Trans. Acoust., Speech, Signal Process., vol. 36, no. 2, pp. 292-294, Feb. 1988.

[33] G. Kadel, "Diversity and equalization in frequency domain a robust and flexible rreceiver technology for broadband mobile communication systems," in Proc. IEEE 47th Vehicular Technology Conf. (VTC), May 4-7, 1997, vol. 2, pp. 894-898.

[34] Channel Models for Fixed Wireless Applications, IEEE Std. 802.16.3c-01/19r4, 2001.

[35] G. L. Stüber, Principles of Mobile Communications. Norwell, MA: Kluwer Academic, 1996. 


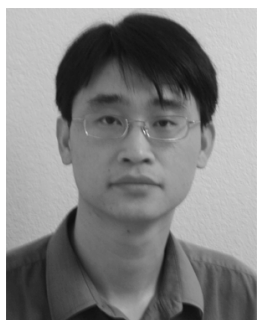

Li Guo received the B.Eng. degree in radio communication engineering from the Beijing University of Posts and Telecommunications, Beijing, in July 1998 and the M.S.E.E. degree from the University of Notre Dame, Notre Dame, IN, in January 2004. He received the Ph.D. degree in electrical engineering from the University of Notre Dame in 2007.

Since April 2006, he has been a Senior Systems Engineer with Navini Networks Inc., Richardson, TX, where he works on broadband wireless access echnologies. His research interests include adaptive signal processing, statistical estimation and detection theory, multiuser and multiantenna system, and broadband wireless access.

Mr. Guo placed second in the Final Student Paper Contest at the 39th Asilomar Conference on Signals, Systems, and Computers, November 2005.

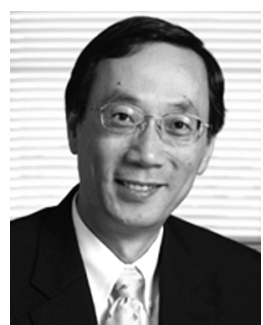

Yih-Fang Huang (F'95) received the B.S.E.E. degree from National Taiwan University, R.O.C., in 1976, the M.S.E.E. degree from the University of Notre Dame, IN, in 1980, and the Ph.D. degree in electrical engineering from Princeton University, Princeton, NJ, in 1982

Since 1982, he has been on the faculty of the University of Notre Dame where he is currently Professor of Electrical Engineering. In spring 1993, he received the Toshiba Fellowship and was Toshiba Visiting Professor at Waseda University, Tokyo, Japan, in the Department of Electrical Engineering. His research interests focus on statistical communications and adaptive signal processing. Collaborating with his students and colleagues, he has developed set-membership filtering (SMF) and identification and derived a group of adaptive algorithms noted as optimal bounding ellipsoids (OBE). His recent interests are in applications of SMF and SMI to multiple-access and wireless communication systems, particularly on issues of interference mitigation.

Dr. Huang was the area editor on Mathematics for the Circuits and Filters Handbook (Boca Raton, FL: CRC /IEEE, 1995) and the area editor on Signal Processing for Electrical Engineering Handbook (New York: Elsevier/ Academic, 2005). He has been on the Editorial Board for the Journal of Franklin Institute. He was an Associate Editor for the IEEE TRANSACTIONS ON CIRCUITS AND SYSTEMS (1989-1991) and Associate Editor for its Express Letters during 1992-1993. He was Vice President for the IEEE Circuits and Systems Society during 1997-1998 and was a Distinguished Lecturer for the same society during 2000-2001. He was awarded the University of Notre Dame's Presidential Award in 2003, and the Golden Jubilee Medal of the IEEE Circuits and Systems Society in 1999 . 